\title{
Complex Dynamics in a Simple Model of Interdependent Open Economies
}

\author{
SHAHRIAR YOUSEFI $^{\mathrm{a}, *}$, YURI MAISTRENKO $^{\mathrm{b}, \dagger}$ and SVITLANA POPOVYCH ${ }^{\mathrm{b}}$ \\ ${ }^{a}$ The Econometric Group, Department of Statistics and Demography, University of Southern Denmark, \\ Main Campus, Odense University, Campusvej 55, 5230 Odense M, Denmark; ${ }^{\mathrm{b}}$ Institute of Mathematics, \\ National Academy of Sciences of Ukraine, Tereschenkivska St. 3, 252601 Kiev, Ukraine
}

(Received 15 March 2000)

\begin{abstract}
Based on a simple two-market model, characterized by a demand link between competitive markets for goods, a system of coupled difference equations is used to represent the interdependent structure of a global economy. Relying on numerical and analytical approaches, various dynamic properties of the proposed model are explored. Among others, a general specification of the regions of stability of the equilibrium and main periodic cycles, the transition to chaos through torus destruction, chaotic synchronization, and the coexistence of different types of attractors in parameter space are described. Typical bifurcation processes are illustrated.
\end{abstract}

Keywords: Competitive two-market model; Stability; Bifurcation; Coexistence of attractors

\section{INTRODUCTION}

The purpose of this paper is to investigate the complicated inherent dynamics of a system of interdependent open economies. In the first phase of the study, a simple interacting cobweb model is introduced. Relying on relatively common assumptions, the model is used to describe the interactive dynamics of two interdependent markets. This model, in its initial form, is defined for a closed economy with no external interaction. Models of this type were previously proposed by
Currie and Kubin (1995) and by Hommes and van-Eekelen (1996) in order to investigate the relevance of application of partial equilibrium analysis in economics.

In the next phase of the study, the framework of the original model is extended in order to address the dynamics of an interdependent global economy. Considering the framework of the global economy and relying on the insight provided by Armington (1969), it is assumed that there is a trade flow among different open economies for goods that are distinguished not only by their

\footnotetext{
*Corresponding author. e-mail: syo@sam.sdu.dk

†e-mail: maistrenko@imath.kiev.ua
} 
technical specifications but also by other characteristics such as their place of origin. A dynamic model derived in the framework of a system of coupled difference equations is consequently proposed as a reasonable representation for the interdependent structure of the global economy.

It is important to emphasize that the underlying reasoning behind the present work is to confine the study to the essentials, i.e., to strip the problem of all the flesh until we are left with the main structure, and no further simplification is possible.

Following this strategy, the focus is directed towards the rich inherent dynamics of the model and towards some of its characteristics that are analytically or numerically tractable.

The model is derived in the general framework of a discrete two-dimensional map. Although it initially looks deceptively simple, it conceals a rich complex dynamics that resemble similar patterns observed in higher dimensional maps. Modern theory of the nonlinear dynamical systems, developed in recent decades, is well suited to shed light on the nature of complex dynamics, although many essential questions still remain open (see, e.g., Palis and Takens, 1993; Shilnikov et al., 2000; Iliashenko and Weigu, 1999).

The proposed model is presented as a twodimensional map, $F$, with an inherent quadratic non-linearity. The fact that $F$ is non-invertible, in other words that it is an endomorphism but not a diffeomorphism, implies that critical curves exist in the phase space $\mathbb{R}^{2}$ where the Jacobian $D F$ is equal to zero. Due to the presence of these two main characteristics of the map $F$ (i.e., non-linearity and noninvertibility) rigorous analytical studies of the global dynamics often lead to significant difficulties. To the best of our knowledge, so far, this kind of system has not been subjected to mathematical investigations at an advanced level. Any such investigation must incorporate two different aspects, namely the global dynamics of diffeomorphisms and the theory of critical lines developed in Mira et al. (1996).

An example of the application of nonlinear twodimensional maps in economics is provided by
Brock and Hommes (1997), and the mechanism of the transition to chaos through homoclinic bifurcations is illustrated. Our example appears to be different. As we have found numerically, the transition to chaos in our model mainly takes place through torus destruction. The theory developed in Newhouse et al. (1983) (see also Shilnikov et al., 2000; Iliashenko and Weigu, 1999) can be applied in this case in order to provide additional analytical insight into the phenomena.

One of the main objectives of the present study is to provide a preliminary understanding of the main dynamic features of the proposed model for the interdependent global economy. With this purpose, the regions of stability for the equilibrium and for the periodic cycles of period 2 in parameter space are analytically specified. Moreover, the further developments in the dynamics of the system are followed numerically when the parameters are varied. It is demonstrated that the evolution of the system typically takes place through a Hopf bifurcation followed by torus destruction and finally a boundary crisis.

A short description of the main features observed in the process of chaotic synchronization of the map is presented. This phenomenon can take place when the two coupled maps are identical.

It is interesting to notice the relatively large regions in parameter space where the phenomenon of multistability, or the coexistence of different attractors, occurs. This phenomenon is of profound significance since the long-term behavior of the system will not only depend on the given parameters, but also on the initial states of the system. With identical sets of parameters and different initial conditions, trajectories can move towards different attractors.

\section{THE TWO-MARKET MODEL}

In order to be self-contained, a brief description of a slightly modified version of the Currie and Kubin (1995) model follows. 
Assume that the economy consists of two markets and that there is no external interaction with other economies. Further more, assume that the first good takes one period to produce and that the corresponding price, $p_{1}(t)$, adjusts instantaneously. The market clearing price is determined by a linear demand function given by

$$
q_{1}(t)=a_{1}-p_{1}(t)+s_{1} p_{2}(t)
$$

and the unit profit of the first good is given by

$$
\pi_{1}(t)=p_{1}(t)-c(1+r)-T_{1}
$$

where $p_{2}(t)$ is the price of the second good, the parameter $a_{1}$ is positive, and the parameter $s_{1}$ is positive (negative) if the goods are substitutes (complements).

The parameter $c$ is the fixed unit cost. $r$ is the fixed interest rate, and $T_{1}$ is the fixed unit tax on the first good.

A simple quantity adjustment process is postulated, assuming that the rate of change in the production is proportional to the unit profit, that is,

$$
\frac{q_{1}(t+1)-q_{1}(t)}{q_{1}(t)}=\sigma \pi_{1}(t) .
$$

For the second market it is assumed that there are linear demand and supply functions, and that the market clears instantaneously. There is no production lag, and, using a similar notation, we obtain the following equations,

$$
\begin{gathered}
q_{2}^{d}(t)=a_{2}+s_{2} p_{1}(t)-p_{2}(t), \\
q_{2}^{s}(t)=b\left(p_{2}(t)-T_{2}\right),
\end{gathered}
$$

and

$$
q_{2}^{s}(t)=q_{2}^{d}(t)
$$

Solving for the quantity of the first good yields the iterative quadratic map

$$
q_{1}(t+1)=\mu q_{1}(t)+\eta q_{1}^{2}(t)
$$

with the substitutions

$\mu=1+\sigma\left(\frac{a_{1}(1+b)+s_{1}\left(a_{2}+b T_{2}\right)}{1+b-s_{1} s_{2}}-c(1+r)-T_{1}\right)$

and

$$
\eta=\sigma \frac{1+b}{1+b-s_{1} s_{2}} .
$$

The map given in Eq. (7) is a variant of the celebrated logistic map. Using a slightly modified notation and the following transformation it can be rewritten in the standard form

$$
x_{t+1}=\mu x_{t}\left(1-x_{t}\right) ; \quad x_{t}=\frac{\eta}{\mu} q_{1}(t) .
$$

Consequently the dynamics of the first good, $q_{1}(t)$, in the economy can be studied through the dynamics of $x_{t}$ and alteration in the space of the parameters of the model.

\section{THE INTERDEPENDENT MODEL}

Analysing the global trade pattern among different economies is often linked to the validity of the socalled "perfect substitutability assumption" of the tradable goods. This assumption simply implies that goods of a given type supplied by sellers in one country are perfect substitutes for the same sort of goods supplied by any other country. A wellknown example of the application of this assumption is the celebrated Hecksher-Ohlin approach (see, e.g., Gandolfo, 1998; Wong, 1995; Markusen and Melvin, 1988; Johns, 1985; Jones, 1979; Ellis and Metzler, 1950; Ohlin, 1933; Heckscher, 1919).

Armington (1969) challenges the validity of this assumption and argues that seemingly perfect substitutable goods (such as French chemicals vs. Japanese chemicals) should be regarded as different products and distinguished specifically in the analysis. 
Challenging the validity of perfect substitutability assumption leads to a different modelling approach in which goods "... are distinguished from one another in the sense that they are assumed to be imperfect substitutes in demand. Not only is each good, such as chemicals, different from any other good but also each good is assumed to be differentiated (from the buyers' viewpoint) according to the suppliers' area of residence" (Armington, 1969).

Over the course of time, there have been other approaches in which the product differentiation is endogenously defined and related to a certain firm and not a country (see Dixit and Stiglitz, 1977). Other approaches relate the presence of product differentiation to a desire for variation of the products among the consumers or alternatively to a certain degree of heterogeneity among the consumers (see Helpman and Krugman, 1989). Besides these topics, it is also desirable to incorporate preferences and other behavioral related peculiarities in the general analysis.

As previously noted, since our main strategy is to confine the problem to the essentials, we choose not to elaborate on these aspects in the present study, but to direct our focus towards the inherent complicated dynamics of the model.

Relying on this understanding, in the next stage of the study, the initial framework of the model is expanded by considering the global economy and its various economies as an interdependent system. This interdependence represents the introduction of trade among open economies. In this way, we intend to contribute to the discussion on partial equilibrium analysis by addressing the global dynamics of the expanded model. In order to do so, a simplified version of the global model is introduced by considering the case with two economies each characterized by similar assumptions and properties (as given by Eqs. (1)-(10)).

This will yield the following system of equations.

$$
\begin{aligned}
& x_{t+1}=\mu_{1} x_{t}\left(1-x_{t}\right) \\
& y_{t+1}=\mu_{2} y_{t}\left(1-y_{t}\right)
\end{aligned}
$$

Introducing trade leads to an interdependence between the economies. Limiting trade to the first good, a simple form of interdependence can be expressed by the following coupled dynamics

$$
\left\{\begin{array}{l}
x_{t+1}=\mu_{1} x_{t}\left(1-x_{t}\right)+\gamma_{1} y_{t} \\
y_{t+1}=\mu_{2} y_{t}\left(1-y_{t}\right)+\gamma_{2} x_{t}
\end{array}\right.
$$

where $\mu_{1,2} \in[0,4]$ and $\gamma_{1,2} \in \mathbb{R}$.

The fact that we propose to deal with a twodimensional process in $\left(x_{t}, y_{t}\right)$ space in the framework outlined in Eq. (12) implies that the dynamics of the first good is no longer solely determined in terms of the local policy parameters (such as unit tax, interest rate, etc.) or local structural parameters (such as unit cost, demand interdependence, etc.). Thus, in contrast to the framework outlined in Eq. (11), using linear coupling as proposed by Eq. (12) facilitate the introduction of a new component in the analysis, namely the interdependent structure of the economy and consequently the trade policy parameters. The coupling coefficients $\gamma_{1}$ and $\gamma_{2}$ can be interpreted as trade policy parameters. Obviously, as long as there is no trade between the economies, $\gamma_{1}=\gamma_{2}=0$. The parameters $\mu_{1}$ and $\mu_{2}$ are defined by Eq. (8) in the framework of the two market economies.

In the next section, the issue of complicated global behavior of this system will be addressed.

\section{CHAOTIC SYNCHRONIZATION AND ASYNCHRONOUS CYCLES}

Consider the simple version of the expanded model with two open economies and restrain the trade to the first good. Consequently, the system of two coupled difference equations given by (12) can provide a reasonable representation for the interdependent structure of the global economy.

Numerical simulations indicate, that the system given by Eq. (12) has many common features with 
the symmetrical case obtained from Eq. (12), in which $\mu_{1}=\mu_{2}=\mu$, and $\gamma_{1}=\gamma_{2}=\gamma$,

$$
\left\{\begin{array}{l}
x_{t+1}=\mu x_{t}\left(1-x_{t}\right)+\gamma y_{t} \\
y_{t+1}=\mu y_{t}\left(1-y_{t}\right)+\gamma x_{t} .
\end{array}\right.
$$

Despite the complicated global behaviour of the symmetrical system, some of its important features appear to be analytically tractable. Due to the presence of symmetry, the system has a onedimensional invariant manifold given by the diagonal $D=\{(x, y): x=y\}$.

Symmetrical motion on the diagonal $D$ is obviously given by the recurrent equation

$$
x_{t+1}=(\mu+\gamma) x_{t}\left(1-x_{t}\right)
$$

and, hence, by the logistic map

$$
f_{a}: x \mapsto a x(1-x), \quad a=\mu+\gamma
$$

The dynamics of the map $f_{a}$ and the corresponding symmetrical behavior of the model given by Eq. (13) may be regular or chaotic (see Collet and Eckmann, 1980), depending on the parameter $a \in[0,4]$. At the same time, the symmetrical behavior (i.e., when $x_{t}=y_{t}$ for all $t$ ) can be stable or unstable with respect to asymmetric perturbations of the initial conditions $\left(x_{0}, y_{0}\right)$.

Transverse stability of the behavior means that the two open economies represented here by the relevant state variables $\left(x_{t}, y_{t}\right)$ can synchronize even if their initial states $\left(x_{0}, y_{0}\right)$ are different.

This property can be expressed by

$$
\left|x_{t}-y_{t}\right| \rightarrow 0 \text { as } t \rightarrow \infty .
$$

The synchronization given by Eq. (16) is called periodic or chaotic, depending on the periodic or chaotic dynamics of the corresponding logistic maps given by Eq. (15). The case of chaotic synchronization is of particular interest. In this case the state variables $x_{t}$ and $y_{t}$ converge towards each other (asymptotically with $t$ ), while the dynamics remains chaotic.
Absence of transverse stability of the symmetrical motion in the diagonal $D$ means that a small asymmetry of the initial states $x_{0}$ and $y_{0}$ can grow with time. Then, in general, there are two scenarios for the desynchronized motion to consider, namely the case in which the trajectories will approach some stable asymmetric regimes, or the case when they eventually return to the synchronizing behavior. Besides this, the asymmetry between $x_{t}$ and $y_{t}$ can also grow until the model collapses.

During the last decade, synchronization of coupled dynamic systems has been the subject of intensive investigations by many scholars, and a variety of applications have been suggested in various fields (see Fujisaka and Yamada, 1983; Pikovsky, 1984; Pikovsky and Grassberger, 1991; Afraimovich et al., 1986; Pecora and Carroll, 1990; Pecora et al., 1997; Ashwin et al., 1996; Kocarev and Parlitz, 1995; Roy and Thoruburg, 1994; Rulkov and Sushchik, 1997; Hasler and Maistrenko, 1997; Ditto and Showalter, 1997; Astakhov et al., 1997; Kapitaniak and Maistrenko, 1999; Yanchuk et al., 2000).

In particular, the system of two coupled identical logistic maps

$$
G:\left(\begin{array}{c}
X \\
Y
\end{array}\right) \rightarrow\left(\begin{array}{c}
a X(1-X)+\gamma(Y-X) \\
a Y(1-Y)+\gamma(X-Y)
\end{array}\right)
$$

in which $a$ and $\gamma$ are real parameters has been investigated by Maistrenko et al. (1998a,b, 1999a, b). This map is topologically conjugated to our map

$$
F:\left(\begin{array}{l}
x \\
y
\end{array}\right)=\left(\begin{array}{l}
\mu x(1-x)+\gamma y \\
\mu y(1-y)+\gamma x
\end{array}\right)
$$

which corresponds to the symmetrical system (13). Indeed, conjugacy between $F$ and $G$ is easily given by the following scale transformation of the state variables:

$$
x=\left(1+\frac{\gamma}{\mu}\right) X, \quad y=\left(1+\frac{\gamma}{\mu}\right) Y,
$$

for $a=\mu+\gamma$. 
Following Maistrenko et al. (1998b), one can numerically identify the region of synchronized behavior in the space of the parameters $(\gamma, \mu)$ for the map $F$. This region is illustrated in Figure 1 and denoted by $\mathcal{R}_{A}$. The points in the region $\mathcal{R}_{A}$ were obtained from the condition of negativeness of the typical transverse Lyapunov exponent $\lambda_{\perp}$ of the symmetrical one-dimensional attractor $A$ situated in the diagonal $D$. The synchronization is chaotic if attractor $A$ is chaotic. This case can clearly take place only in the parameter band $3.569 \cdots<\mu+\gamma \leq 4$ and if the value of the parameter $a=\mu+\gamma$ does not belong to any of the infinitely many windows of periodicity of $f_{a}$ given by Eq. (15).

Actually, the region illustrated in Figure 1 is a region of so-called weak or Milnor stability of the attractor $A$ (see Milnor, 1985). Weak stability guarantees that the attractor $A$ is stable in average, i.e., almost all trajectories on the chaotic set are transversely stable, and so the basin of attraction of $A$ has positive Lebesgue measure in $\mathbb{R}^{2}$. When $A$ is a periodic attractor, weak stability clearly coincides with usual Lyapunov stability. But the weakly stable chaotic attractor $A$ may not be Lyapunov stable.

This particular case in which the chaotic attractor $A$ is weakly stable but not Lyapunov stable is interesting for the structure of the basin of attraction of $A$. In this case, the basin appears to be riddled (see Alexander et al., 1992), i.e., densely filled with points from which the trajectories are not attracted to $A$. The transition to a riddled basin is determinated by the first orbit on the chaotic attractor $A$, which becomes transversely unstable and is referred to as the riddling bifurcation (see Lai et al., 1996).

The riddling bifurcation leads to the appearance of trajectories which are repelled from the attractor $A$ in the transverse direction. Consider trajectories that are originally repelled from the neighbourhood of $A$. The basin is globally riddled if the dynamics of the system allows direct access for the trajectories to go to some other (asynchronous) attractor or infinity. Globally riddled basins resemble the morphology of a fat fractal set with a maximal density concentrated around the attractor $A$ as well as around the preimages of $A$.

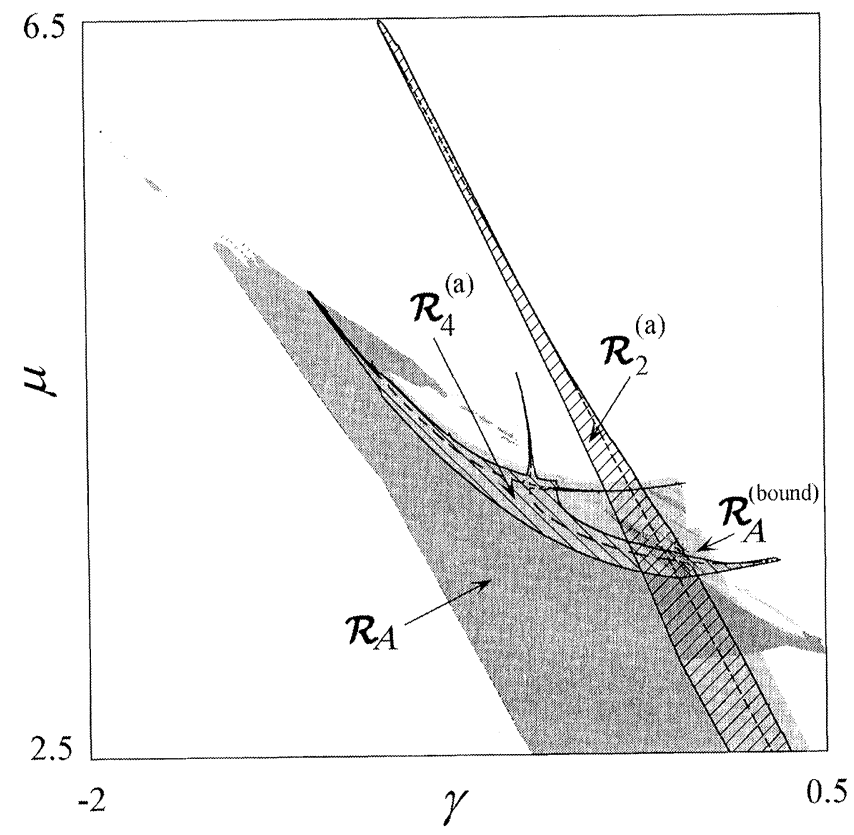

FIGURE 1 Parameter regions for typical dynamical regimes of the system (13). Details are specified in the text. 
Alternatively, if there is no access for the trajectories to go to other attractors or infinity, it is typical for allmost all trajectories to return to the neighborhood of $A$, and consequently, by repeating such behavior, bursts away from the diagonal are produced. At the end, most of the trajectories will be eventually attracted to $A$. In this case, the basin of $A$ appears to be only locally riddled (see Ashwin et al., 1996; Maistrenko et al., 1997; Maistrenko et al., 1999b). It is filled with initial conditions that are not leading to $A$, but the set that consists of all of these initial conditions is characterized by having a Lebesgue measure of zero.

Hence, the detailed structure of locally riddled basins can not be observed by standard computational procedures, but by reliance on more specific approaches (see Pikovsky and Grassberger, 1991).

It was previously reported by Maistrenko et al., (1998a) and by Bischi and Gardini (1998) that the distinction between these two types of riddling processes depends mostly on the existence of socalled absorbing and mixed absorbing areas (see Mira et al., 1996). These regions of state space derive from the theory of two-dimensional noninvertible maps (see Mira et al., 1996). They control to a large extent the global dynamics of the system given by (13), and in many cases they restrain trajectories starting near the synchronized chaotic attractor $A$ from reaching other limiting sets or infinity.

Riddling bifurcation curves belonging to the region $\mathcal{R}_{A}$ are determined by Maistrenko et al. (1998b) (see Fig. 5 in that paper).

Region $\mathcal{R}_{A}$ represents parameter combinations that lead (at least) to a weak stability of $A$. The boundaries of $\mathcal{R}_{A}$ consist of the parameter points $(\gamma, \mu)$ for which the typical transverse Lyapunov exponent $\lambda_{\perp}$ of the attractor $A$ changes its sign from negative to positive, and the so-called blowout bifurcation takes place for the map $F$ (see Ott and Sommerer, 1994). After the blowout bifurcation, an invariant chaotic set $A$ in the diagonal $D$ still exists, but it is transformed into a so-called chaotic saddle (see Nusse and Yorke,
1991). Only a zero-measure set of the trajectories are attracted to it, so they are not detectable by regular numerical procedures. Special procedures in which chaotic saddles can be obtained have been proposed by Nusse and Yorke (1998). Nevertheless, due to the finite precision of calculations, one can observe that trajectories eventually end up in the chaotic saddle $A$, even when the transverse Lyapunov exponent $\lambda_{\perp}$ is slightly positive.

Consider the stable asynchronous regimes which are dominating in the model (13). Apparently there are two such dominating regimes characterized by asynchronous period- 2 and asynchronous period-4 motions.

Depending on the parameters, each of the motions can be either regular (in which case the attractor is a point cycle or a piece wise ergodic torus), or chaotic (in which case the attractor is piecewise chaotic). Parameter regions for the stability of the period-2 and period-4 motions are shown in Figure 1 as dashed regions and denoted by $\mathcal{R}_{2}^{(a)}$ and $\mathcal{R}_{4}^{(a)}$, respectively.

Asynchronous period-2 and period-4 point cycles $\left(P_{2}^{(a)}\right.$ and $P_{4}^{(a)}$ ) have emerged via a transverse period-doubling bifurcation of the symmetrical fixed point $P_{1}=\left(x^{*}, x^{*}\right)$ and the symmetrical period-2 cycle $P_{2}^{(s)}=\left(\left(x_{1}, x_{1}\right), \quad\left(x_{2}, x_{2}\right)\right)$, respectively. In the next phase $P_{2}^{(a)}$ and $P_{4}^{(a)}$ lose their stability in a Hopf bifurcation. Curves for the Hopf bifurcations are shown by dashed lines inside $\mathcal{R}_{2}^{(a)}$ and $\mathcal{R}_{4}^{(a)}$ (for details, see the next section of the paper).

After the Hopf bifurcation, a closed invariant curve (called also torus) emerges with a quasiperiodic motion on it. This is followed by a periodic motion on the torus. Later on, the torus loses its smoothness, and the destruction of the torus will occur due to the further variations in the space of parameters. This process typically leads to a strange attractor (Newhouse et al., 1983, see also Shilnikov et al., 2000; Iliashenko and Weigu, 1999) which, after a number of transformations, vanishes in a boundary crisis (upper right boundary of the regions in Figure $1 \mathcal{R}_{2}^{(a)}$ and $\mathcal{R}_{4}^{(a)}$ ). 

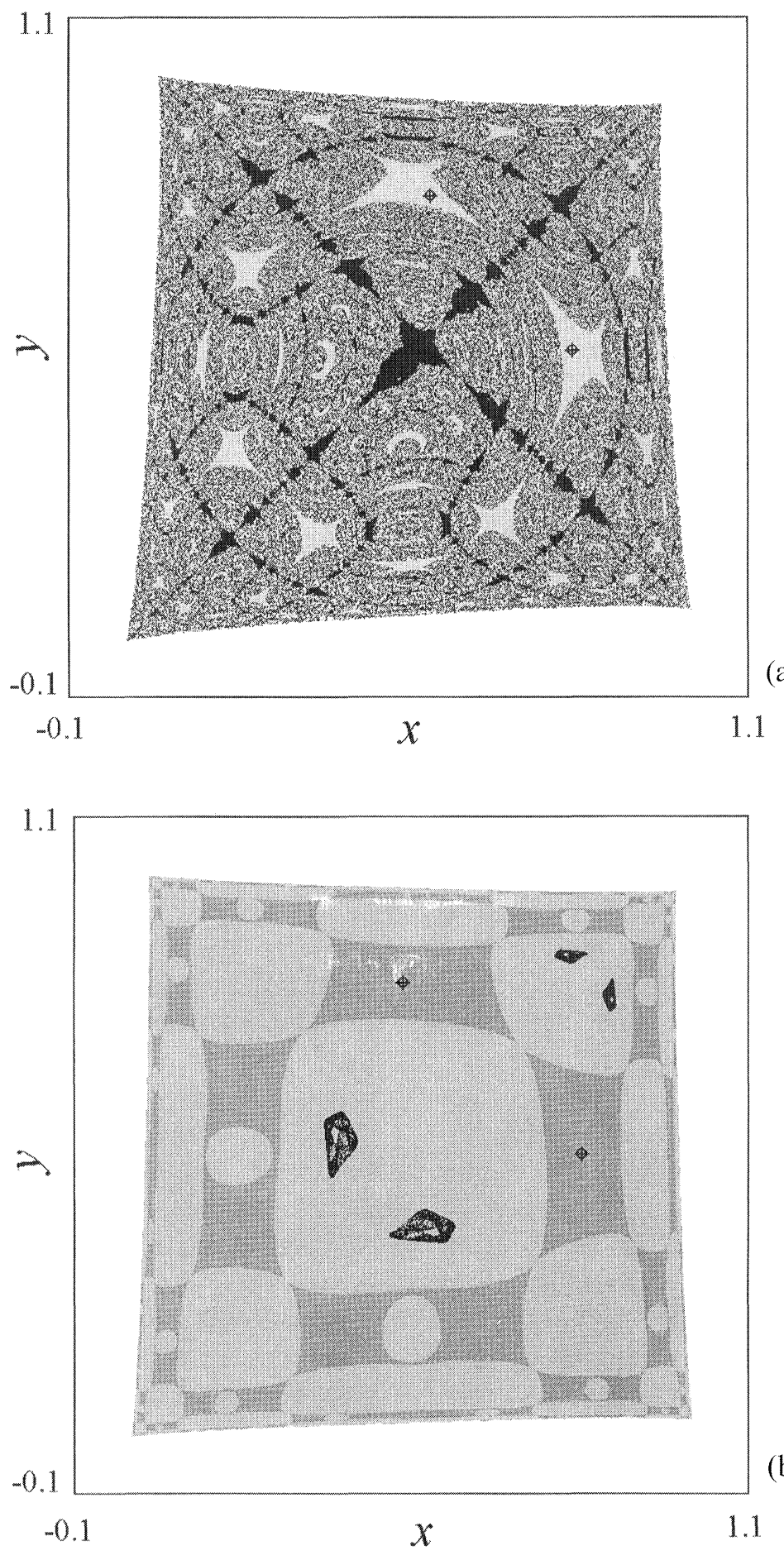

FIGURE 2 Basins structure of the system (13) for different values of the parameters $\mu$ and $\gamma:$ (a) coexistence of asymmetrical period-2 stable cycle (basin is grey) and symmetrical 4-piece chaotic attractor in the diagonal (basin is black), at $\mu=3.77$ and $\gamma=$ -0.2 ; (b) coexistence of two asymmetrical attractors: period-2 stable cycle (basin is dark grey) and 4-piece chaotic attractor (basin is light grey), at $\mu=3.6$ and $\gamma=-0.105$; (c) coexistence of three attractors: symmetrical period- 8 stable cycle (basin is dark grey), asymmetrical period-2 invariant curve, i.e., torus (basin is grey) and asymmetrical 4-piece chaotic attractor (basin is light grey), at $\mu=3.59$ and $\gamma=-0.035$; (d) two-dimensional chaotic attractor of the system (13), at $\mu=2.8$ and $\gamma=0.31$; (basin is light grey). In all examples, the basin of infinity is left blank. 

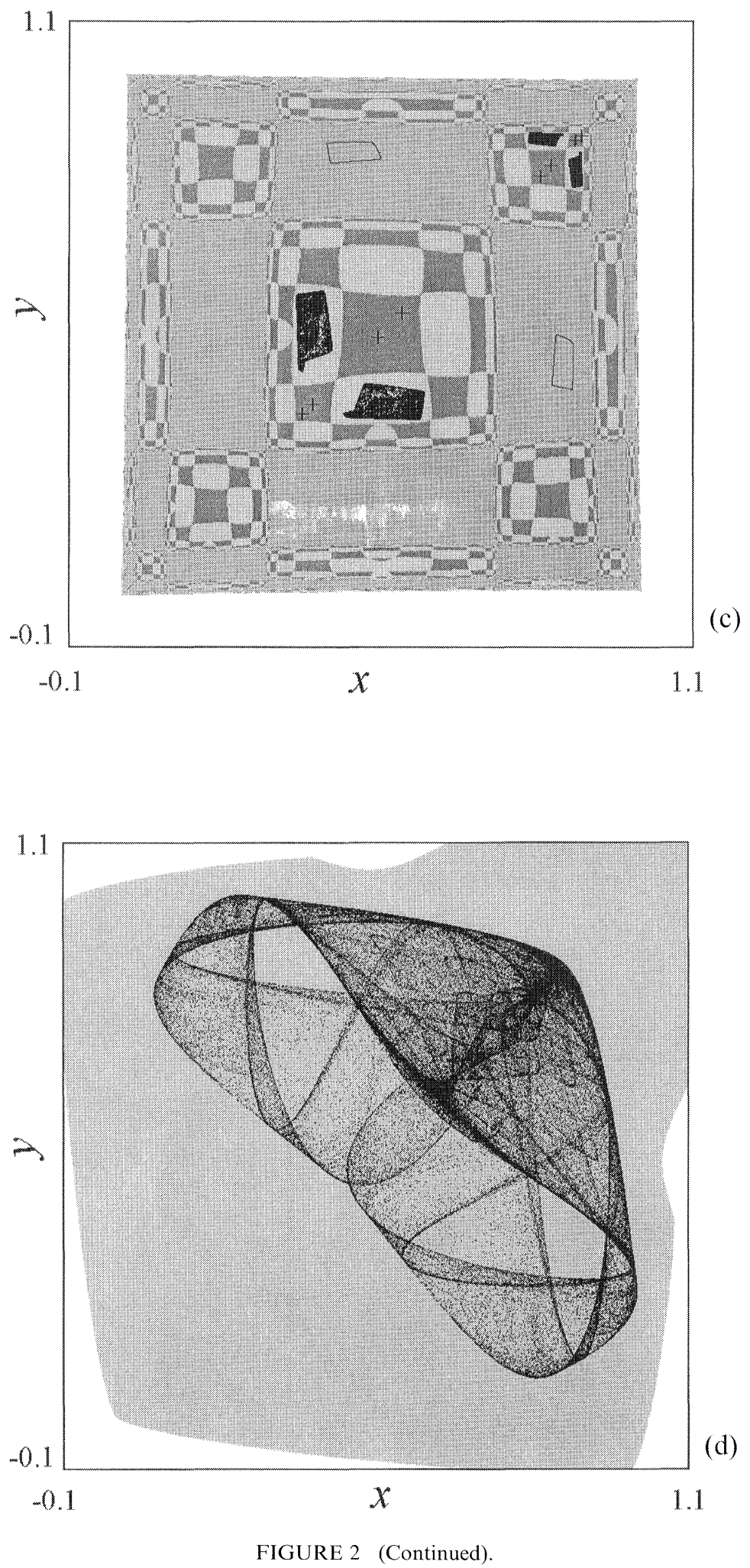
Many essential features that are usually occurring with the cycle $P_{2}^{(a)}$ and $P_{4}^{(a)}$ appear to be similar. But the shape of the parameter region $\mathcal{R}_{4}^{(a)}$ is clearly different from $\mathcal{R}_{2}^{(a)}$ since $\mathcal{R}_{4}^{(a)}$ apparently has a characteristic form similar to a "shrimp" (a typical stability region for two-dimensional maps). It is caused by the fact that in addition to the cycle $P_{4}^{(a)}$, other stable period- 4 cycles can exist inside $\mathcal{R}_{4}^{(a)}$.

It is important to underline that although there are infinitely many other regions of stability of other asynchronous attractors of $F$, the set of parameters for these regions appears to be of a relatively insignificant size. Further elaboration on these points is omitted in the present paper.

Therefore, we conclude that there are predominantly three regions of stability in the parameter plane $(\gamma, \mu)$ for the map $F$, characterized as regions $\mathcal{R}_{2}^{(a)}$ and $\mathcal{R}_{4}^{(a)}$ for asynchronous attractors, and region $\mathcal{R}_{A}$ for, the symmetrical attractors placed on the diagonal.

As illustrated in Figure 1, it is interesting to notice the intersection of these three regions in the space of parameters. In other words, there is a rather significant region in the parameter plane in which two or even three of the attractors coexist.

This observation leads to an important issue concerning the relative structure of the basins of attraction of these attractors. One can begin with the case in which, starting from the different initial conditions, eventually leads to the realization of different asymptotic regimes. Examples of different basin structures are presented in Figure 2. Among possible scenarios the case in which one of the regimes is synchronous but the others are not (case a) can be mentioned. Besides that, the case in which both regimes are asynchronous, but one is regular (periodic or quasiperiodic) and the other chaotic (case b) can be mentioned. Moreover, the case in which even three of these attractors coexist (case c) can be imagined.

This interesting property leads to the conclusion that, given the same combination of parameters, it is conceivable that there are, roughly speaking, three different scenarios concerning the patterns of behavior for the dynamics of the system, all depending on the initial conditions.

This property is of particular significance for economic theory. Our simple model illustrates that, due to the inherent dynamics of the interdependent global economy, identical structural policies (represented by the same combination of parameters in the two-markets model) and trade policies (represented by coupling parameters) can eventually lead to different regimes of behavior, all depending on the initial states of the economies (for further discussion see Section 6).

Furthermore, one particular region is of essential significance in studying the asynchronous dynamics of $F$. This region contains all points in the space of parameters $(\gamma, \mu)$ of the model (13) for which the trajectories when starting near the attractor $A$ are bounded. This region is denoted by $\mathcal{R}_{A}^{\text {(bound) }}$ and visualized in light grey in Figure 1 . $\mathcal{R}_{A}^{\text {(bound) }}$ intersects the above regions $\mathcal{R}_{A}, \mathcal{R}_{2}^{(a)}$ and $\mathcal{R}_{4}^{(a)}$, but none of them belongs to it. Consider those parameter values $(\gamma, \mu) \in \mathcal{R}_{A}^{\text {(bound) }}$ that do not belong to any of the previously mentioned regions $\mathcal{R}_{A}, \mathcal{R}_{2}^{(a)}$ and $\mathcal{R}_{4}^{(a)}$. Then, as numerical simulations indicate, there is a rather large probability that the attractor of the map $F$ will be two-dimensional and in such a way that the one-dimensional invariant chaotic set $A$ belongs to it. In Figure 2d, an example of this kind of attractor is presented for the parameter values $\mu=2.8$ and $\gamma=0.31$.

\section{STEADY STATE AND PERIOD-2 POINT CYCLES}

In the present part of our study, the focus is directed towards the behavior of the equilibrium, for the symmetrical version of the proposed model for the global economy given by Eq. (18).

There are (at most) four conceivable fixed points for the map denoted as $F$ given by Eq. (18). Among them are the two symmetrical points on the diagonal $D$ (i.e. $(0,0)$ and $\left(x^{*}, x^{*}\right)=((\mu+\gamma-1) /$ $\mu,(\mu+\gamma-1) / \mu))$ and two asymmetrical points 
$\left(x_{1}^{*}, y_{1}^{*}\right)$ and $\left(x_{2}^{*}, y_{2}^{*}\right)$, given by:

$$
\begin{aligned}
x_{1,2}^{*} & =y_{2,1}^{*} \\
& =\frac{1}{2 \mu}(\mu-\gamma-1 \pm \sqrt{(1+\gamma-\mu)(1-\mu-3 \gamma)}) .
\end{aligned}
$$

The asymmetrical fixed points exist for the following ranges of the parameters

$$
\begin{aligned}
& 1-3 \gamma<\mu<1+\gamma \quad \text { if } \mu>1, \\
& 1+\gamma<\mu<1-3 \gamma \quad \text { if } \mu<1 .
\end{aligned}
$$

Asymmetrical fixed points do not contribute much to the inherent dynamics of the model since they can never be stable and do not give rise to any other more complicated attractors of the map $F$.

The important case relates to the symmetrical fixed points which can stabilize in some regions of parameter space. Moreover, when losing stability, they initiate more complicated stable regimes. Let us consider this issue in more details.

Diagonal $D$ is invariant with respect to the map $F$. In other words, starting from any initial point $\left(x_{0}, y_{0}\right) \in D$, the initiated trajectory never leaves $D$ under the action of $F$. Therefore, the dynamics in the diagonal is clearly given by the one-dimensional quadratic map

$$
f_{\mu, \gamma}: x \mapsto-\mu x^{2}+(\mu+\gamma) x
$$

which for $x=(1+\gamma / \mu) X$ and $a=\mu+\gamma$ reduces to the logistic map $f_{a}$ given by Eq. (15).

Assuming $f_{a}=f_{\mu+\gamma}$, the fixed point $(0,0)$ is stable in the parameter range $-1<\mu+\gamma<1$, while the fixed point $\left(x^{*}, x^{*}\right)$ is stable for $1<$ $\mu+\gamma \leq 3$. At $\mu+\gamma=3$, this second fixed point undergoes a period-doubling bifurcation that leads to a stable period- 2 cycle. Reaching the parameter value, $\mu+\gamma=1$ corresponds to a transcritical bifurcation which interchanges the stability between the fixed points $(0,0)$ and $\left(x^{*}, x^{*}\right)$.

Therefore, in the parameter range $-1<$ $\mu+\gamma \leq 3$, the logistic map given by Eq. (15) has one stable fixed point, $x^{*}$, which is equal to
0 , if $\mu+\gamma<1$ and equal to $(\mu+\gamma-1) / \mu$, if $\mu+\gamma>1$.

In order to obtain the region of stability of the equilibrium $P_{1}=\left(x^{*}, x^{*}\right)$ of the two-dimensional map $F$, the focus is directed towards the longitudinal eigendirection (i.e., along the diagonal $D$ ) of $P_{1}$ and the transversal eigendirection which is perpendicular to $D$.

The corresponding eigenvalues are consequently denoted by

$$
\nu_{\|}=f^{\prime}\left(x^{*}\right)=2-\mu-\gamma(\text { parallel })
$$

and

$$
\nu_{\perp}=f^{\prime}\left(x^{*}\right)=2-\mu-3 \gamma(\text { transversal }) .
$$

The stability of the fixed point $P_{1}$ is conditioned on the following inequalities

$$
\left\{\begin{array}{l}
1-\gamma<\mu<3-\gamma \\
1-3 \gamma<\mu<3-3 \gamma
\end{array}\right.
$$

since both eigenvalues in this case will lie inside the unit circle.

These inequalities provide the region of stability of $P_{1}$ in the space of parameters of the model, denoted by $\mathcal{R}_{1}$ in Figure 3 for positive values of $\mu$. Besides $\mathcal{R}_{1}$, the stability region of period- 2 cycles $P_{2}^{(s)}$ and $P_{2}^{(a)}$ are also visualised, denoted as $\mathcal{R}_{2}^{(s)}$ and $\mathcal{R}_{2}^{(a)}$, respectively.

Each of these cycles $\left(P_{2}^{(s)}\right.$ and $\left.P_{2}^{(a)}\right)$ emerges from $P_{1}$ in a period-doubling bifurcation. But for $P_{2}^{(s)}$ it happens in the bifurcation which goes along the diagonal, and so $P_{2}^{(s)} \in D$ and is specified by:

$$
\begin{aligned}
P_{2}^{(s)}=\{ & \left(x_{1}^{(s)}, x_{1}^{(s)}\right),\left(x_{2}^{(s)}, x_{2}^{(s)}\right) \in D: x_{1,2}^{(s)} \\
& \left.=\frac{\mu+\gamma+1 \pm \sqrt{(\mu+\gamma+1)(\mu+\gamma-3)}}{2 \mu}\right\} .
\end{aligned}
$$

The bifurcation curve for the synchronous period-doubling bifurcation of $P_{1}$ is given by

$$
\mathcal{L}^{(s)}=\{(\gamma, \mu): \mu=3-\gamma\}
$$




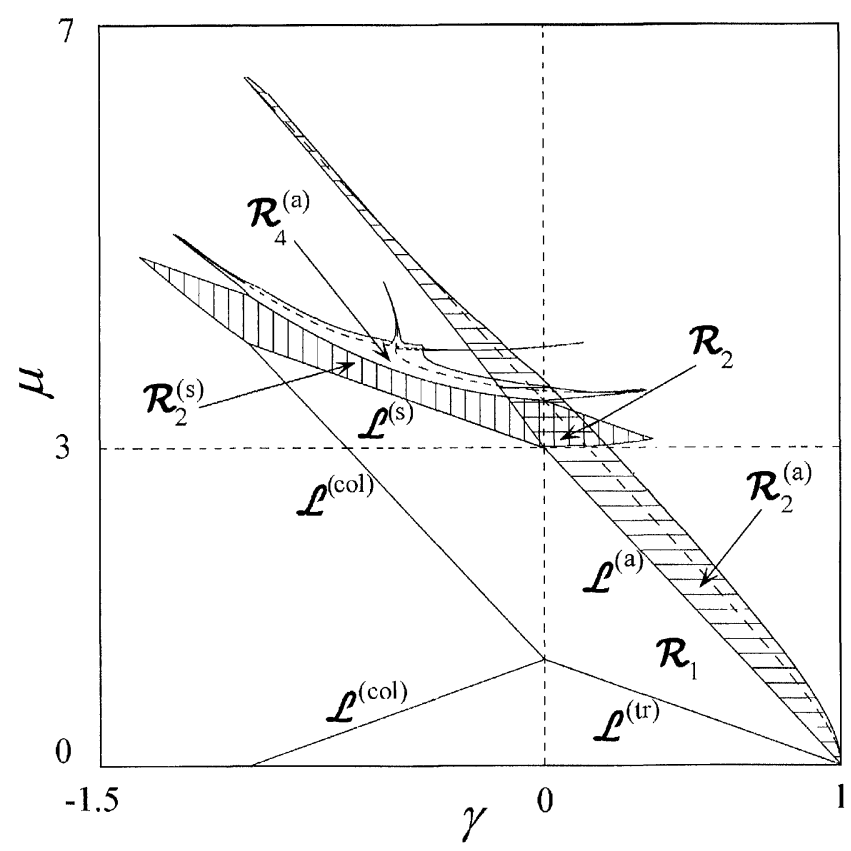

FIGURE 3 Parameter regions for the stability of the equilibrium $\left(\mathcal{R}_{1}\right)$, symmetrical period-2 cycle $\left(\mathcal{R}_{2}^{(s)}\right)$, asymmetrical period-2 cycle $\left(\mathcal{R}_{2}^{(a)}\right)$, and asymmetrical period-4 cycle $\left(\mathcal{R}_{4}^{(a)}\right)$ of the system (13). Details are specified in the text.

which is obtained from the condition $\nu_{\|}=-1$. The curve denoted by $\mathcal{L}^{(s)}$ serves as a boundary between the regions $\mathcal{R}_{1}$ and $\mathcal{R}_{2}^{(s)}$.

Another bifurcation curve, $\mathcal{L}^{(a)}$, is obtained from the condition $\nu_{\perp}=-1$. This corresponds to the transversal period-doubling bifurcation of $P_{1}$ and is given by;

$$
\mathcal{L}^{(a)}=\{(\gamma, \mu): \mu=3-3 \gamma\}
$$

The curve denoted by $\mathcal{L}^{(a)}$ serves as a boundary between $\mathcal{R}_{1}$ and $\mathcal{R}_{2}^{(a)}$.

Cycle $P_{2}^{(a)}$ is situated out of the diagonal and is defined as

$$
\begin{aligned}
& P_{2}^{(a)} \\
& =\left\{\left(x_{1}^{(a)}, y_{1}^{(a)}\right),\left(x_{2}^{(a)}, y_{2}^{(a)}\right): x_{1,2}^{(a)}=y_{2,1}^{(a)}\right. \\
& \left.\quad=\frac{\mu+\gamma+1 \pm \sqrt{(\mu+\gamma-1)^{2}-4(\gamma-1)^{2}}}{2 \mu}\right\} .
\end{aligned}
$$

The left side boundary of the steady state region $\mathcal{R}_{1}$ (denoted by $\mathcal{L}^{(\text {coll) }}$ in Fig. 3) corresponds to a pitchfork bifurcation of $P_{1}$, in which the transverse eigenvalue $\nu_{\perp}$ leaves the unit circle through the point +1 .

This pitchfork bifurcation appears to be subcritical and does not give rise to any new stable cycle or another attracting state.

Moreover, in the present case, this bifurcation characterizes a tendency towards the collapse of the system. In other words, after that, when the parameters are chosen to the left of $\mathcal{L}^{\text {(col) }}$, there are no other attractors in the whole phase space $\mathbb{R}^{2}$. And consequently, all the trajectories that do not belong to the diagonal diverge towards infinity. Another interesting bifurcation curve which lies inside the region $\mathcal{R}_{1}$ is denoted by $\mathcal{L}^{(\mathrm{tr})}$ in Figure 3. This curve visualizes the emergence of transcritical bifurcation of the fixed point $P_{1}$. Below $\mathcal{L}^{(\text {tr) }}$, the steady state $P_{1}$ is trivial and equal to $(0,0)$, while above it, the steady state $P_{1}$ is non-trivial and equal to $\left(x^{*}, x^{*}\right)$. Given $(\gamma, \mu) \in \mathcal{L}^{(\text {tr })}$, the fixed point $P_{1}$ is only stable from one side. 
As illustrated in Figure 3, the stability regions $\mathcal{R}_{2}^{(s)}$ and $\mathcal{R}_{2}^{(a)}$ of the synchronous and asynchronous cycles share a rather large intersection given by $\mathcal{R}_{2}=\mathcal{R}_{2}^{(s)} \cap \mathcal{R}_{2}^{(a)}$ in the space of parameters. It is interesting to observe that for $(\gamma, \mu) \in \mathcal{R}_{2}$ the stable synchronous and stable asynchronous period-2 regimes coexist and, depending on the initial conditions $\left(x_{0}, y_{0}\right)$, a given trajectory will tend either to $P_{2}^{(s)}$ or to $P_{2}^{(a)}$ as $t \rightarrow \infty$. The trajectory can also go to infinity if the initial point $\left(x_{0}, y_{0}\right)$ lies out of both the basins.

Figure 4 presents examples of basins structures for the cases when the parameter point is in $\mathcal{R}_{1}$ (case a), $\mathcal{R}_{2}^{(s)}$ (case b), $\mathcal{R}_{2}^{(a)}$ (case c) and $\mathcal{R}_{2}=$ $\mathcal{R}_{2}^{(s)} \cap \mathcal{R}_{2}^{(a)}$ (case d), respectively. The basin of $\mathcal{R}_{2}^{(s)}$ is marked in dark grey, the basin of $\mathcal{R}_{2}^{(a)}$ in light grey, and the basin of the set of points diverging to the infinity is left blank.

Particularly, for the intersection region $\mathcal{R}_{2}$, we can see that if the initial conditions $\left(x_{0}, y_{0}\right)$ are chosen near the diagonal $D$, the trajectory goes, with a rather large probability, towards the synchronous period-2 cycle $P_{2}^{(s)}$. Otherwise, if the initial conditions $\left(x_{0}, y_{0}\right)$ are chosen away from the diagonal (but not too far), there is a larger probability that the trajectory moves towards the asynchronous period-2 cycle $P_{2}^{(a)}$. Increasing the

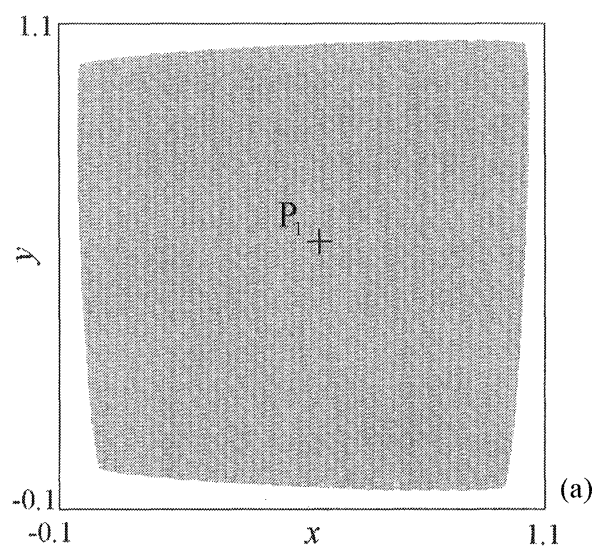

FIGURE 4 Basins structure of the system (13) for different values of the parameters $\mu$ and $\gamma:$ (a) $(\gamma, \mu) \in \mathcal{R}_{1}$, at $\mu=2$ and $\gamma=0.1$; (b) $(\gamma, \mu) \in \mathcal{R}_{2}^{(s)}$, at $\mu=3.3$ and $\gamma=-0.1$; (c) $(\gamma, \mu) \in \mathcal{R}_{2}^{(a)}$, at $\mu=2.9$ and $\gamma=0.1$; (d) $(\gamma, \mu) \in \mathcal{R}_{2}$, at $\mu=3.1$ and $\gamma=0.1$. Points of corresponding cycles are marked by cross.
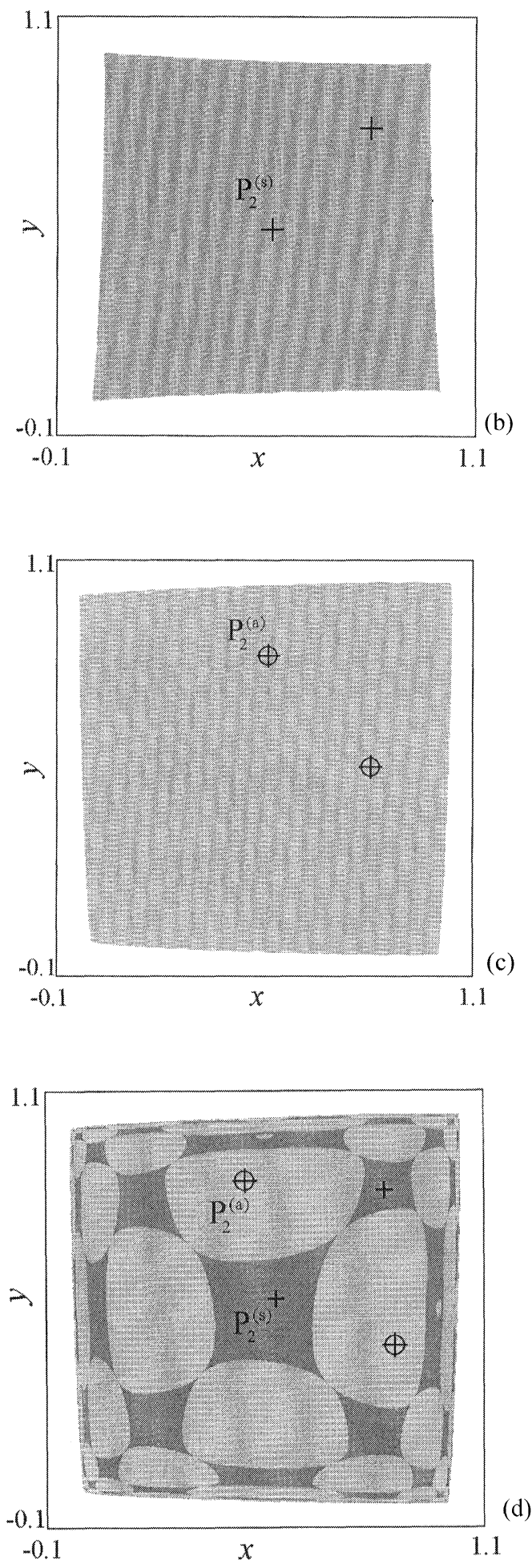

FIGURE 4 (Continued). 


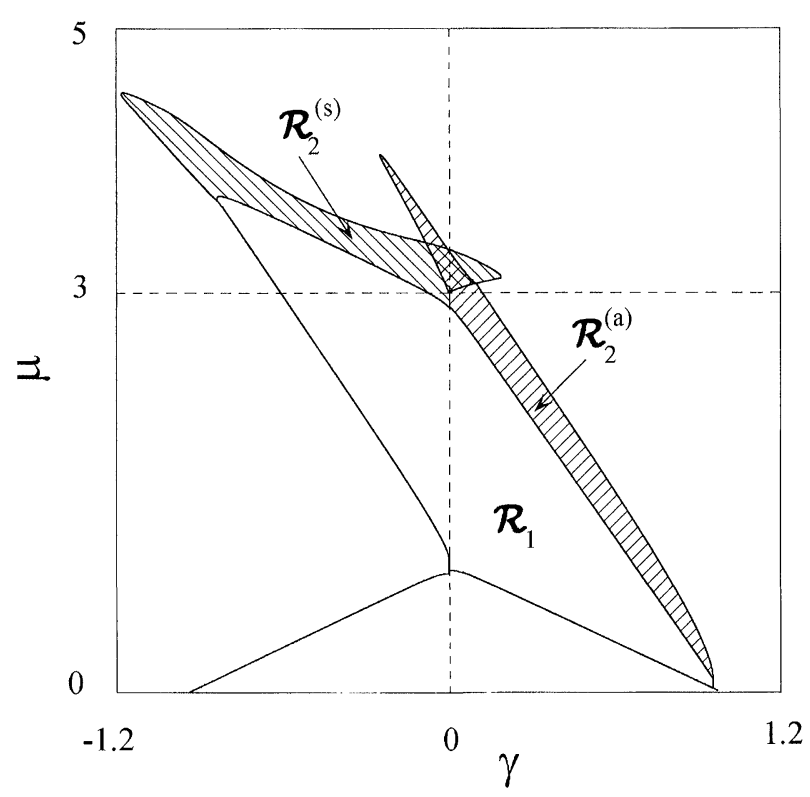

FIGURE 5 Parameter regions for the stability of the equilibrium, and the symmetrical and asymmetrical period-2 cycles of the systems (12), where $\mu_{1}=\mu, \mu_{2}=\mu_{1}+\varepsilon, \gamma_{1}=\gamma_{2}=\gamma$, at $\varepsilon=0.1$.

distance between $x_{0}$ and $y_{0}$ will eventually lead to the divergence of the trajectory towards infinity.

Another interesting peculiarity of the shape of the parameter regions in Figure 3 is that they spread into the domain $\mu>4$. This phenomena occurs for negative values of the coupling coefficient $\gamma$. In order to provide a more visual interpretation, consider the uncoupled case $(\gamma=0)$ of the model given by Eq. (13) and let parameter $\mu$ assume a value more than 4, e.g., $\mu \approx 4.5$. Then almost all trajectories of Eq. (13) diverge. But, with a decreasing coupling coefficient, the system will initially stabilize, first to the asymmetric cycle $P_{2}^{(a)}$ and then to the symmetric cycle $P_{2}^{(s)}$ before it finally becomes diverging again.

\section{DISCUSSIONS}

The standard trade models are often different variations of the so-called Computable General Equilibrium (CGE) family of models (for some recent studies, Karunaratne, 1998; Rattso and Torvik, 1998; Chang, 1997; Rodrigo and Martin,
1997; Harrison et al., 1997; Smith and Spinosa, 1997).

Despite the popularity of these types of models, there is little evidence to suggest that these models do possess an inherent ability to provide significant insight into the general topology of the policy space. This is due to the fact that these models are predominantly designed in a comparative statics framework. From an epistemological viewpoint, this property constitutes the main critique for using these classes of models for studying trade flows. One practical remedy is to link the model to a dynamic macro model, but such interventions can only provide a second-hand impression of the dynamics of trade. This problem is especially significant from a dynamic modelling approach when different policy mixes can be represented by specific points in the parameters space of the model.

Therefore, it is reasonable to ask whether such an approach is appropriate for analysing a volatile process such as the global trade dynamics.

On the other hand, in economics there is a tradition of modelling dynamic processes, 
especially in discrete time. The Cournot duopoly model and the Samuelson-Hicks business cycle model are among the well-known examples of this tradition (for further references, see Puu, 1997 and Lorenz, 1989).

Inspired by this tradition, we have formulated the presented interdependent model of global economy. Despite the simple structure of the model, so far, we have come a long way in this paper. In very few words, we have illustrated how a simple global model of trade characterized by a demand link between competitive markets for goods and a linear trade coupling between economies can be represented by a non-invertible system of coupled difference equations with an inherent non-linearity. Reaching this point enabled us to conduct various numerical and analytical investigations that eventually provided a deeper understanding of a number of interesting features of the model.

Among these features were the observation that the transition to chaos mainly takes place through a torus destruction, and a general specification of the regions of stability for the main equilibrium and for the point cycles of period 2 and 4 in the parameter space. Moreover, it was demonstrated that the evolution of the system typically involves a Hopf bifurcation followed by torus destruction and, finally, a boundary crisis. This was followed by elaborations on the emergence of the process of chaotic synchronization in the model including a short description of the process. Later on, the existence of the phenomenon of multistability, or the coexistence of different attractors in the parameter space, was demonstrated.

Besides the mathematical significance of the presented results, these results can also be appreciated from an economic viewpoint. One particular view concerns the policy design and the related implications of different policy measures. This issue was dealt with by exploring the general topology of policy space, since different policy mixes were represented by specific points in the parameters space of the model.

As previously mentioned, our model focuses on real trade flows and incorporates a certain degree of insulation (or interdependence) of domestic markets from the world markets. The demand links (incorporated as determinants of $\mu$ defined in Eq. (8)) and particularly the linear couplings provide the mechanism through which the dynamics is transmitted across markets and economies. Following this approach, the consequences of structural adjustment programs and macroeconomic policy packages can be reflected into the space of parameters of the global model (i.e., the $(\gamma, \mu)$ space). Therefore, specification of the regions of stability of the equilibrium in parameter space corresponds to a general classification of the qualitative behavior of the global model when economies are subject to different policy mixes and their related measures.

Moreover, the existence of the phenomenon of multistability or the coexistence of different attractors in the parameter space corresponds to the case in which even identical policies designed for identical economies can not guarantee similar behavior.

However, it should be mentioned that we obtain these results at the cost of not including many details and specific modelling of different factors such as the exchange rate, labour markets, capital markets, etc., so the model in the presented form should be regarded as a prototype of a more detailed modelling approach.

Finally, we would like to make some brief remarks on another interesting feature observed in the model. This issue will be addressed specifically and in more details in future. For the time being we only consider the mathematical framework of the model and omit further elaborations on interdependences between the parameters of the original two-markets model and parameters (and variables) of the system of coupled difference equations. The focus is then solely directed towards analytically tractable characteristics of the symmetrical system of two coupled one-dimensional quadratic maps given by Eq. (13).

Consider the asymmetrical system (12) and set $\mu_{1}=\mu, \mu_{2}=\mu+\varepsilon, \quad \gamma_{1}=\gamma$, and $\gamma_{2}=\gamma+\delta$. The parameters $\varepsilon$ and $\delta$ characterize the magnitude of 
the mismatches between $\mu_{1}$ and $\mu_{2}$, and between $\gamma_{1}$ and $\gamma_{2}$, respectively.

In Figure 5, the bifurcation structure of the system (13) is illustrated, given $\varepsilon=0.1$ and $\delta=0$. As visualized in this figure, although regions of stability of the steady state and of the synchronous and asynchronous period- 2 cycles undergo deformations, their shapes remain quite similar to the original ones plotted in Figure 3 for the symmetrical system (12).

Numerical simulations indicate that the evolution of the system (12) from regular to chaotic is going on through the same "torus destruction" scenario which (as described in Section 5) controls the complications of the dynamics for the symmetrical system (12). Therefore, small mismatches do not distort the period-2 and period-4 asynchronous regimes. Moreover, these regimes are seemingly maintaining their dominance in the asymmetrical system (12). This phenomenon also concerns the two-dimensional chaotic attractors which can typically exist for the parameter values $(\gamma, \mu)$ belonging to the region $\mathcal{R}^{\text {(bound) }}$.

At the same time, the chaotic synchronization behavior characterized by Eq. (16) will cease to exist at any small mismatch. This is due to the fact that the diagonal $D$ is no longer invariant. Moreover, seemingly there are no longer any one-dimensional invariant manifolds for the system (12) if $\mu_{1} \neq \mu_{2}$ and (or) $\gamma_{1} \neq \gamma_{2}$. This leads to an important question concerning the further developments of the symmetrical chaotic attractor $A$ when the parameters are subject to alteration (i.e., mismatches are conceivable).

As it was pointed out in Popovych et al. (2000) for the analogous system with nonlinear coupling, small mismatches can transform $A$ into a twodimensional chaotic attractor placed around $A$. This is apparently due to the existence of absorbing and mixed absorbing areas enveloping $A$. If such an area exists and does not contain any other attracting states, then, with small mismatches, it can become a chaotic attractor for the asymmetrical system (12).

\section{Acknowledgments}

We thank Gustav Kristensen and Anna Lise Kianzad for discussions and comments on product differentiation. We also thank Vladimir Maistrenko and Olexander Popovych for a number of illuminating discussions and assistance with numerical computations. Yuri Maistrenko and Svitlana Popovych express their gratitude to the staff and collegues at the Department of Statistics and Demography, University of Southern Denmark for their hospitality during preparation of this paper.

\section{References}

Afraimovich, V. S., Verichev, N. N. and Rabinovich, M. I. (1986) General synchronization. Izv. Vyssh. Uch. Zav. Radiofizika, 29, 795-803.

Alexander, J. C., Yorke, J. A. and You, Z. (1992) Riddled basins. Int. J. Bif. Chaos., 2, 795-813.

Armington, P. S. (1969) A theory of demand for products distinguished by place of production. International Monetary Fund Staff Papers, 16, 159-178.

Ashwin, P., Buescu, J. and Stewart, I. (1996) From attractor to chaotic saddle: A tale of transverse instability. Nonlinearity, 9, $703-737$

Astakhov, V., Shabunin, A., Kapitaniak, T. and Anischenko, V. (1997) Loss of chaos synchronization through the sequence of bifurcations of saddle periodic orbits. Phys. Rev. Lett., 79, 1014-1017.

Bischi, C. I. and Gardini, L. (1998) Role of invariant and minimal absorbing areas in chaos synchronization. Phys. Rev. E, 58, 5710-5719.

Brock, W. A. and Hommes, C. H. (1997) A rational route to randomness. Econometrica, 65, 1059-1095.

Chang, S. I. (1997) The Effects of Economic Integration between North and South Korea: A Computable General Equilibrium Analysis. Int. Econ. J., 11(4), 1-16.

Collet, P. and Eckmann, J. P. (1980) Iterated maps on the interval as dynamical systems. Birkhauser, Basel, p. 248.

Currie, M. and Kubin, I. (1995) Non-linearities and partial analysis. Econ. Lett., 49, $27-31$.

Ditto, W. L. and Showalter, K. (1997) Introduction: Control and synchronization of chaos. Chaos., 7, 509-511.

Dixit, A. K. and Stiglitz, J. E. (1977) Monopolistic competition and optimum product diversity. Am. Econ. Rev., 67, 297-308.

Ellis, H. S. and Metzler, L. A. (Eds.) (1950) American Economic Association Readings in the Theory of International Trade. Richard D. Irwin, Inc., Homewood, Ill.

Fujisaka, H. and Yamada, T. (1983) Stability theory of synchronized motion in coupled-oscillator systems. Progr. Theor. Phys., 69, 32-47.

Gandolfo, G. (1998) International trade theory and policy. Springer-Verlag. 
Harrison, G. W., Rutherford, T. F. and Tarr, D. G. (1997) Economic Implications for Turkey of a Customs Union with the European Union. Eur. Econ. Rev., 41(3-5), 861-70.

Hasler, M. and Maistrenko, Yu. (1997) An introduction to the synchronization of chaotic systems: Coupled skew tent maps. IEEE. Trans. CS - I: Fund. Theor. Appl., 44, 856-866.

Heckscher, E. (1919) The effect of foreign trade on the distribution of income. Ekonomisk Tidskrift, 21, 1-32.

Helpman, E. and Krugman, P. E. (1989) Trade policy and market structure. MIT-Press.

Hommes, C. and van-Eekelen, A. (1996) Partial equilibrium analysis in a noisy chaotic market. Econ. Lett., 53, 275-282.

Iliashenko, Yu. and Weigu, Li. (1999) Nonlocal Bifurcations (Mathematical Surveys and Monographs, N 66). Am. Math. Soc., Portland.

Johns, R. A. (1985) International trade theories and the evolving international economy. Frances Pinter Publishers.

Jones, R. W. (1979) International trade: essays in theory. NorthHolland.

Kapitaniak, T. and Maistrenko, Yu. (1999) Riddling bifurcations in coupled piecewise linear maps. Physica D, 126, $18-26$

Karunaratne, N. D. (1998) Macroeconomic Insights on the Liberalised Trading Regime of Thailand. Int. J. Soc. Econ., 25(6-7-8), 1142-59.

Kocarev, L. and Parlitz, U. (1995) General approach to chaotic synchronization with applications to communication. Phys. Rev. Lett., 74, $5028-5031$.

Lai, Y. C., Grebogi, C., Yorke, J. A. and Venkataramani, S. C. (1996) Riddling bifurcation. Phys. Rev. Lett., 77, 55-58.

Lorenz, H. W. (1989) Nonlinear dynamical economics and chaotic motion. Springer-Verlag.

Maistrenko, Yu., Kapitaniak, T. and Szuminski, P. (1997) Locally and globally riddled basins in two coupled onedimensional maps. Phys. Rev. E, 56, 6393-6399.

Maistrenko, Yu., Maistrenko, V., Popovich, A. and Mosekilde, E. (1998a) Role of the absorbing area in chaotic synchronization. Phys. Rev. Lett., 80, 1638-1641.

Maistrenko, Yu., Maistrenko, V., Popovich, A. and Mosekilde, E. (1998b) Transverse instability and riddled basins in a system of two coupled logistic maps. Phys. Rev. E, 57, $2713-2724$.

Maistrenko, Yu., Maistrenko, V., Popovych, O. and Mosekilde, E. (1999a) Desynchronization of chaos in coupled logistic maps. Phys. Rev. E, 60, 2817-2830.

Maistrenko, Yu., Maistrenko, V., Popovych, O. and Mosekilde, E. (1999b) Unfolding of the riddling bifurcation. Phys. Lett. A, 262, 355-360.

Markusen, J. R. and Melvin, J. R. (1988) The theory of international trade. Harper and Row.

Milnor, J. (1985) On the concept of attractor. Commun. Math. Phys., 99, 177-195.

Mira, C., Gardini, L., Barugola, A. and Cathala, J.-C. (1996) Chaotic dynamics in two-dimensional noninvertible maps. World Scientific, Singapore, p. 607.
Newhouse, S., Palis, J. and Takens, F. (1983) Bifurcations and stability of families of diffeomorphisms. Publ. Math. I.H.E.S., 57, 5-71.

Nusse, H. E. and Yorke, J. A. (1991) Analysis of procedure for finding numerical trajectories close to chaotic saddle hyperbolic sets. Ergodic Theor. and Dyn. Sys., 11.

Nusse, H. E. and Yorke, J. A. (1998) Dynamics: Numerical explorations. Applied Mathematical Scinces, 101, Springer, 610.

Ohlin, B. (1933) Interregional and international trade. Harvard University Press.

Ott, E. and Sommerer, J. C. (1994) Blowout bifurcation: The occurrence of riddled basins and on-off intermittency. Phys. Lett. A, 188, 39-47.

Palis, J. and Takens, F. (1993) Hyperbolicity and sensitive chaotic dynamics at homoclinic bifurcations. Cambridge: Cambridge University Press. p. 234.

Pecora, L. and Carroll, T. (1990) Synchronization in chaotic systems. Phys. Rev. Lett., 64, 821-824.

Pecora, L., Carrol, T., Johnson, G., Mar, D. and Heagy, J. (1997) Fundamentals of synchronization in chaotic systems, concepts, and applications. Chaos, 7, 625-643.

Pikovsky, A. S. (1984) On the interaction of strange attractors. Z. Physik B, 55, 149-154.

Pikovsky, A. S. and Grassberger, P. (1991) Symmetry breaking bifurcation for coupled chaotic attractors. J. Phys. A, 24, $4587-4597$

Popovych, O., Maistrenko, Yu., Mosekilde, E., Pikovsky, A. and Kurth, J. Transcritical loss of synchronization in coupled chaotic systems. Physics Letter A (to appear).

Puu, T. (1997) Nonlinear economic dynamics. 4th Edition. Springer-Verlag.

Rattso, J. and Torvik, R. (1998) Zimbabwean Trade Liberalisation: Ex Post Evaluation. Cambridge J. Econ., 22(3), $325-46$.

Rodrigo, G. C. and Martin, W. (1997) Can the World Trading System Accommodate More East Asian Style Exporters? Int. Econ. J., 11(4), 51-71.

Roy, R. and Thoruburg, K. S. (1994) Experimental synchronization of chaotic lasers. Phys. Rev. Lett., 72, 2009-2012.

Rulkov, N. F. and Sushchik, M. M. (1997) Robustness of synchronized chaotic oscillations. Int. J. Bif. Chaos., 7, $625-643$

Shilnikov, L. P., Shilnikov, A. L., Turaev, D. V. and Chua, L. O. (2000) Method of Qualitative Theory in Nonlinear Dynamics - II. World Scientific, Singapore.

Smith, V. K. and Espinosa, J. A. (1997) Environmental and Trade Policies: Some Methodological Lessons. Env. Dev. Econ., 1(1), 19-40.

Wong, K. (1995) International trade in goods and factor mobility. MIT Press.

Yanchuk, S., Maistrenko, Y., Lading, B. and Mosekilde, E. (2000) Effects of a parameter mismatch on the synchronization of two coupled chaotic oscillators. Int. J. Bif. Chaos., 10(3). 


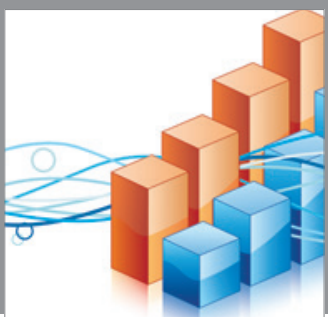

Advances in

Operations Research

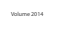

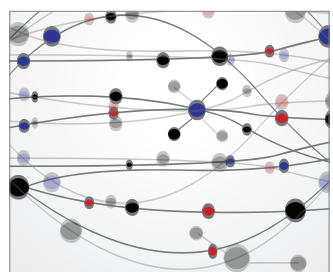

\section{The Scientific} World Journal
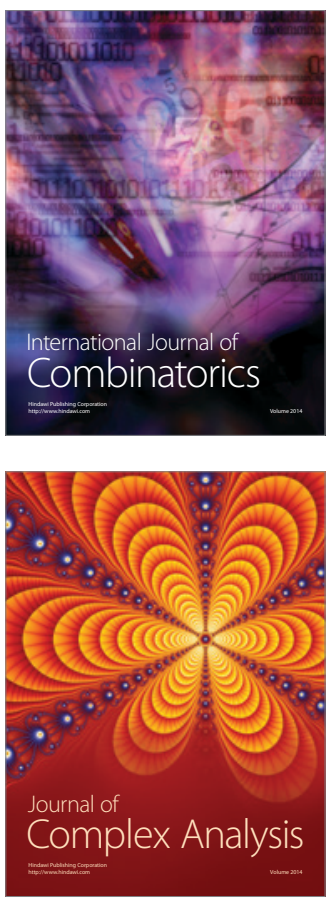

International Journal of

Mathematics and

Mathematical

Sciences
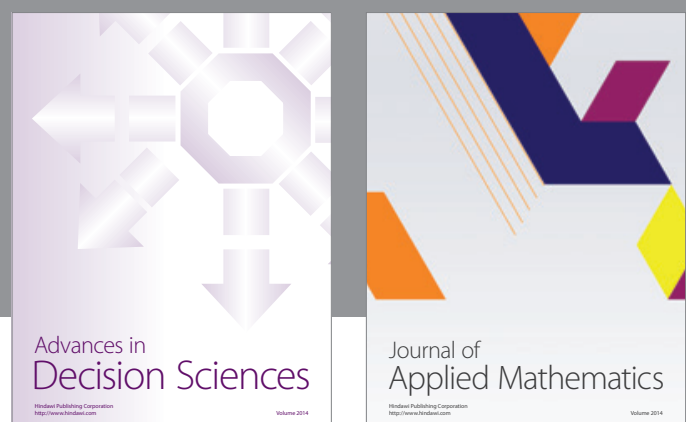

Journal of

Applied Mathematics
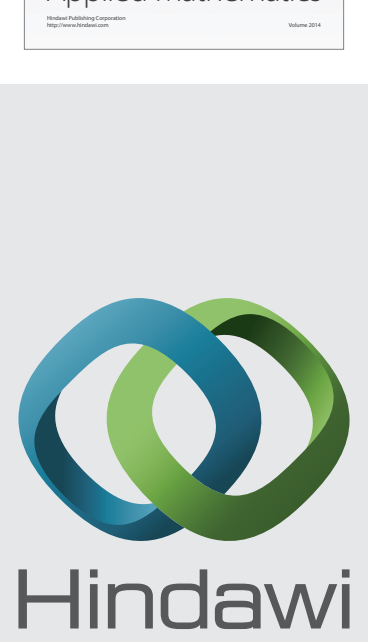

Submit your manuscripts at http://www.hindawi.com
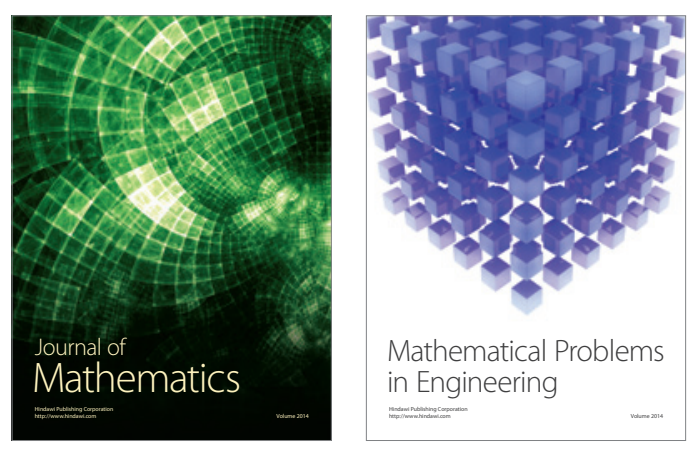

Mathematical Problems in Engineering
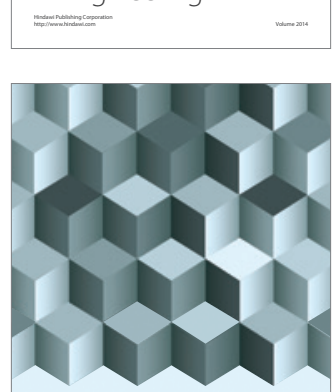

Journal of

Function Spaces
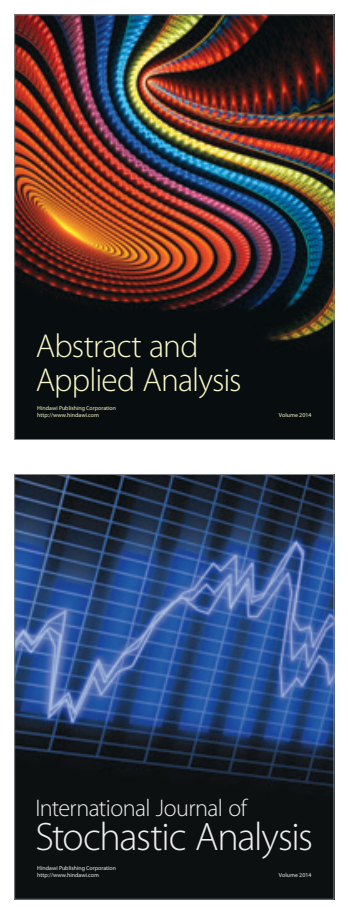

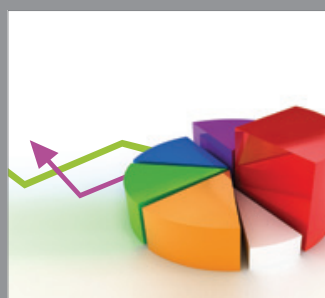

ournal of

Probability and Statistics

Promensencen
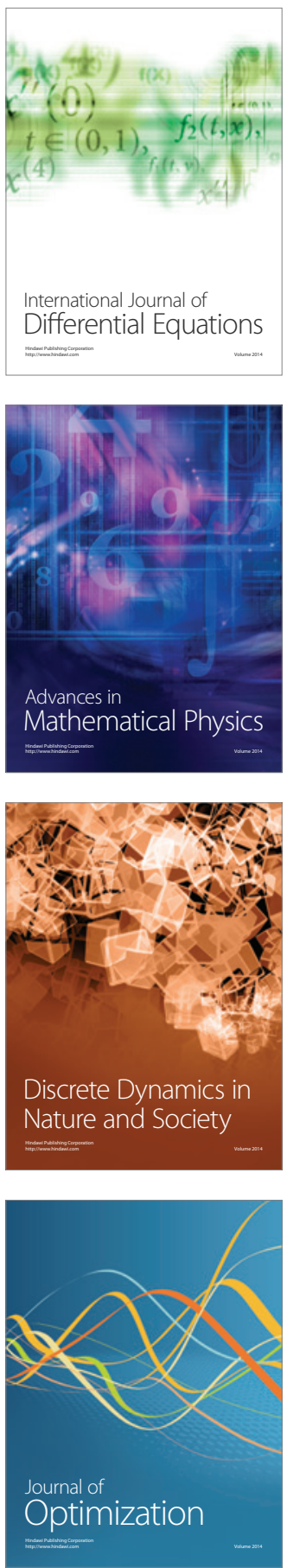